Departamento

de Enfermedades

Respiratorias.

2Departamento de

Anatomía Patológica.

Facultad de Medicina,

Pontificia Universidad

Católica de Chile.

aAlumno de Medicina,

Escuela de Medicina,

Pontificia Universidad

Católica de Chile.

Recibido el 28 de agosto de 2012, aceptado el 27 de noviembre de 2012.

Correspondencia a:

Dr. Arturo Morales Soto.

Departamento

de Enfermedades

Respiratorias.

Facultad de Medicina,

Pontificia Universidad

Católica de Chile.

Marcoleta 350 - Primer

Piso.

Santiago, Chile.

Teléfono: 3543242 /

Celular: 09-5099041

Fax: 6335255

E-mail: ajmorals@gmail.

com

\section{Neumonía en organización por radiación en el paciente con cáncer de mama. Caso clínico}

\author{
ARTURO MORALES SOTO ${ }^{1}$, CARLOS CALVO DE LA BARRA ${ }^{\mathrm{a}}$, \\ GRAÇA PINHEIRO DE CARVALHO ${ }^{1}$, SERGIO GONZÁLEZ BOMBARDIERE², \\ FERNANDO SALDÍAS PEÑAFIEL ${ }^{1}$
}

\section{Radiation organizing pneumonia. Report of one case}

We report a 64 years-old woman who underwent sparing mastectomy with adjuvant radiotherapy for breast cancer. One month after the end of radiotherapy, she presented with malaise, fever, fatigue, cough and migratory bilateral pulmonary infiltrates on serial radiological images. The microbiological studies of broncho alveolar lavage were negative. The patient underwent a trans bronchial biopsy and the pathological diagnosis was compatible with an organizing pneumonia presumably associated with radiotherapy. Systemic steroid treatment was successful with rapid and complete resolution of clinical and radiographic manifestations.

(Rev Med Chile 2013; 141: 664-668).

Key words: Breast neoplasms; Neoplasms; Pneumoniae; Radiation effects.
$\mathrm{L}$ a neumonía en organización (OP) es una entidad clínico-patológica que se manifiesta por malestar general, fatigabilidad, disnea, tos y fiebre, asociada a focos de condensación pulmonar y opacidades en vidrio esmerilado en parches, migrantes en imágenes radiográficas seriadas $^{1}$. En el estudio histopatológico del pulmón se observa tejido de granulación con proliferación de fibroblastos en el lumen de alvéolos y conductos alveolares ${ }^{2}$. La OP puede manifestarse en el contexto de una infección viral, bacteriana o micótica, reacción adversa a drogas, enfermedades reumatológicas, neoplásicas, o bien puede ocurrir sin una causa conocida denominándose criptogénica ${ }^{3}$. Se describe el caso de una paciente con cáncer de mama sometida a radioterapia y que desarrolló una OP.

\section{Caso clínico}

Mujer de 64 años, ex-fumadora de 20 paq/año, con antecedentes de hipertensión arterial crónica en tratamiento con losartán 50 mg/día, con cán- cer de mama izquierda ductal in situ con focos de microinvasión, sometida a una mastectomía parcial con radioterapia coadyuvante de 50 Gy con campos tangenciales, fraccionadas en 25 sesiones de 2 Gy/sesión. Un mes posterior al término de la radioterapia consultó por malestar general, decaimiento, tos irritativa con escasa secreción purulenta, disnea de medianos esfuerzos, dolor dorsal bilateral y fiebre intermitente cuantificada hasta $37,7^{\circ} \mathrm{C}$. En el examen pulmonar se auscultaban crepitaciones finas en la base del hemitórax derecho y anterior izquierdo. En su hemograma destacaba anemia normocítica normocrómica leve (hematocrito: $31 \%$ ) y velocidad de eritrosedimentación (VHS) de $140 \mathrm{~mm} / \mathrm{h}$. La radiografía de tórax (Figura 1A) complementada con una tomografía computada de tórax (TAC) (Figura 1C) mostraba extensos focos de condensación en el lóbulo superior izquierdo y en los lóbulos medio e inferior derecho.

Los exámenes microbiológicos (Gram y cultivo de expectoración, hemocultivos y panel viral) fueron negativos, excepto la medición de inmuno- 
Neumonía en organización por radiación - A. Morales Soto et al

globulina M sérica para Mycoplasma pneumoniae, por lo que fue tratada con levofloxacino $500 \mathrm{mg} /$ día durante diez días, persistiendo en siguientes meses con síntomas y parámetros inflamatorios séricos elevados (VHS: $113 \mathrm{~mm} / \mathrm{h}$ y proteína C reactiva sérica (PCR): $16 \mathrm{mg} / \mathrm{dL}$ (valor normal $<1 \mathrm{mg} / \mathrm{dL})$ ). Una nueva TAC, efectuada a los dos meses y medio de la primera, demostró resolución parcial de los infiltrados pulmonares con aparición de nuevos focos de condensación. El estudio inmunológico con anticuerpos antinucleares y anti DNA, perfil ENA y factor reumatoideo fueron normales. En sus pruebas de función pulmonar destacaban una espirometría normal, capacidad de difusión de monóxido de carbono corregida por hemoglobina (DLCOc) de $14,8 \mathrm{~mL} / \mathrm{min} / \mathrm{mmHg}$ (correspondiente al 90\% del valor teórico) con un volumen alveolar (VA) de 3,6 L (68\% del teórico) y caída significativa de la saturación de pulso de oxígeno $\left(\mathrm{SpO}_{2}\right)$ desde $94 \%$ en reposo a $90 \%$ al fi- nalizar la prueba de caminata de 6 min (DR6), con una distancia recorrida dentro de límites normales.

El cuadro clínico-radiográfico era sugerente de una $\mathrm{OP}$ secundaria por lo que se realizó una fibrobroncoscopia con lavado broncoalveolar (LBA) y biopsias transbronquiales a los 10 días tras última TAC. Los cultivos microbiológicos aeróbicos cuantitativos, de Koch y de hongos del LBA resultaron negativos. El recuento celular diferencial del LBA demostró $90 \%$ de macrófagos, $6 \%$ de linfocitos, $3 \%$ de neutrófilos y menos de $1 \%$ de eosinófilos. La biopsia transbronquial demostró una neumonía intersticial focal con tapones alveolares laxos e infiltrado linfohistiocitario intersticial (Figura 2), compatible con el diagnóstico de OP. Se inició tratamiento esteroidal con prednisona $0,6 \mathrm{mg} / \mathrm{kg} /$ día con descenso gradual progresivo en el plazo de un año. Al cuarto mes de tratamiento tuvo resolución completa de los síntomas y signos clínicos, de las alteraciones radiográficas (Figura 1B), de
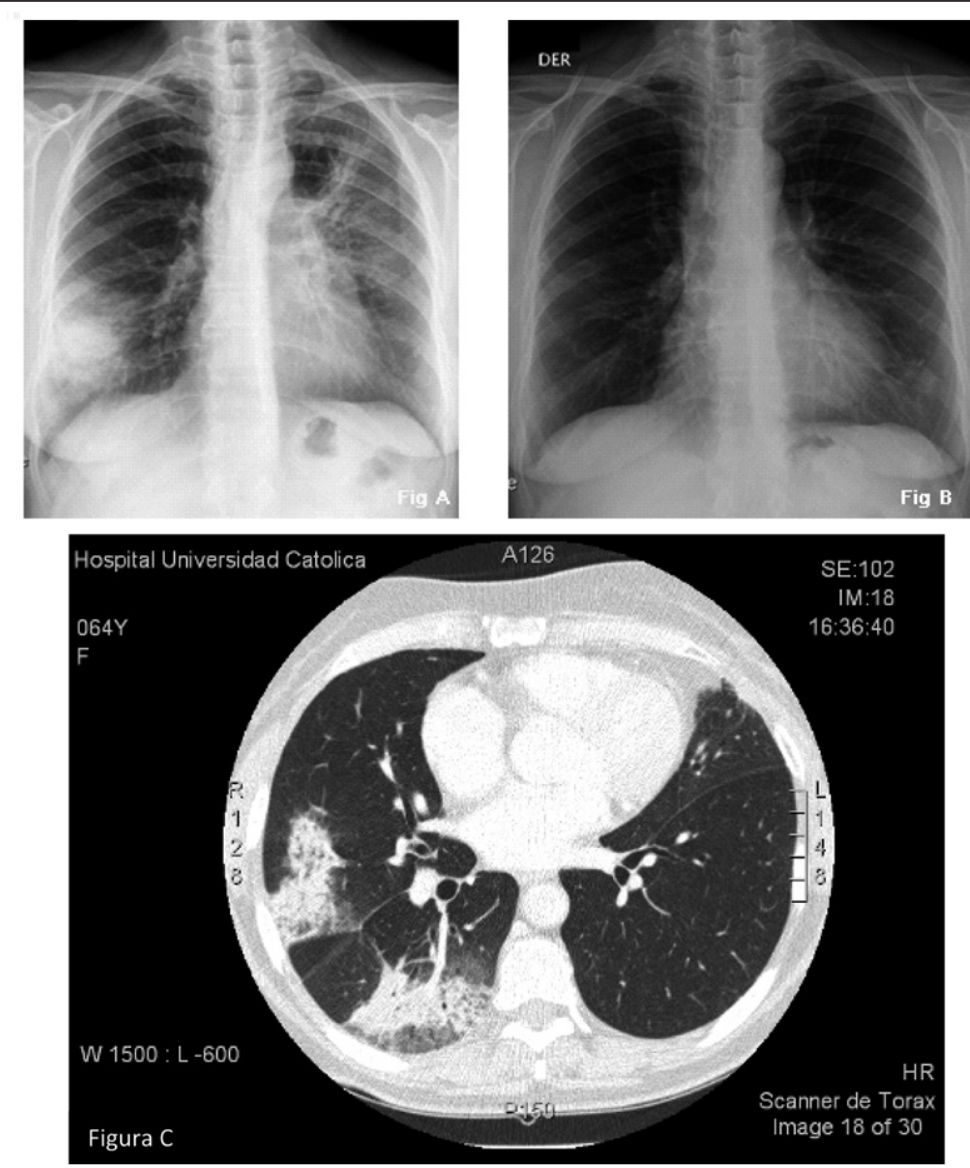

Figura 1. Imágenes pulmonares de paciente con neumonía en organización secundaria a radioterapia. Radiografía de tórax póstero anterior (1A) al inicio del cuadro con focos de condensación en el lóbulo inferior derecho y tenues opacidades en el lóbulo superior izquierdo, que se resuelven a los cuatro meses de tratamiento con esteroides sistémicos (1B). Tomografía computada de tórax en que se observan focos de condensación con áreas de vidrio esmerilado periféricas en el segmento apical del lóbulo inferior derecho y segmento lateral del lóbulo medio, antes de iniciar tratamiento esteroidal (1C). 

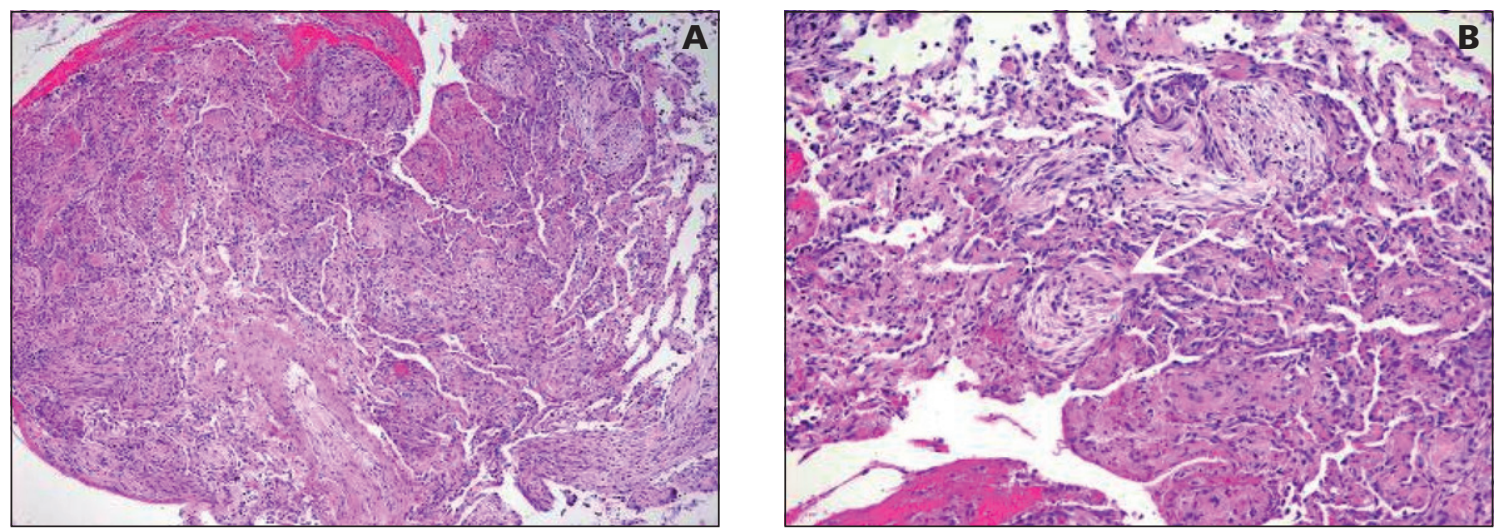

Figura 2. Biospia transbronquial. Microfotografía que muestra tejido pulmonar con tapones intraalveolares de tejido fibroso laxo (cuerpos de Masson señalado por flecha) e infiltrado linfohistiocitario intersticial. Tinción de HE, 100X (A) y 200x (B)

los hallazgos del hemograma y normalización de las pruebas de función pulmonar, con una DLCOc de $16,6 \mathrm{~mL} / \mathrm{min} / \mathrm{mmHg}(100 \%$ del teórico), VA de $4,4 \mathrm{~L}$ ( $83 \%$ del teórico) y $\mathrm{SpO}_{2}$ basal de $97 \%$ y 96\% al final de la DR6.

\section{Discusión}

La radioterapia juega un papel fundamental en el tratamiento del cáncer de mama, permitiendo en estadios iniciales realizar cirugías conservadoras con reducción del riesgo de recidiva tumoral local $^{4}$. La OP asociada a la radioterapia adyuvante del cáncer de mama fue descrita por primera vez en el año 1995 por Crestani y Bayle ${ }^{7,8}$, siendo la incidencia reportada en la literatura entre 1,8\% y $2,5 \% \%^{5,9-11}$. Esta complicación puede afectar el tejido pulmonar más allá de los márgenes de radiación ${ }^{11-13}$, llegando a veces a comprometer el pulmón contralateral ${ }^{9,11,13}$; lo cual no acontece en la neumonitis actínica.

Crestani y cols ${ }^{12}$ describieron las características clínicas, radiológicas y de la función pulmonar en 15 pacientes con OP secundaria a radioterapia por cáncer de mama. Los síntomas principales son disnea y tos seca por más de dos semanas, fiebre intermitente hasta $38^{\circ} \mathrm{C}$ en la mayoría de los casos ${ }^{9}$, pudiendo asociarse astenia, baja de peso y dolor torácico ${ }^{12}$. En el examen pulmonar destacan crepitaciones finas difusas y en los exámenes sanguíneos la elevación de la VHS (entre 45 y $140 \mathrm{~mm} / \mathrm{h}$ ) ${ }^{12}$ y de la PCR ${ }^{9,14}$. Estos hallazgos han sido confirmados en estudios posteriores ${ }^{5,-11}$. Los hallazgos radiográficos y tomográficos más frecuentes son focos de condensación multifocales con áreas de vidrio esmerilado, hallazgos que característicamente migran en imágenes subsecuentes ${ }^{14}$. El tiempo promedio transcurrido entre el término de la radioterapia y la presentación de la OP es de 4 a 8 meses ${ }^{9,10,14,15}$. En las pruebas de función pulmonar se describen patrones espirométricos restrictivos leves a moderados, con disminución leve de la capacidad de difusión de monóxido de carbono e hipoxemia discreta ${ }^{12}$. En todos los pacientes sometidos a radioterapia por cáncer de mama es esperable encontrar un aumento del recuento de linfocitos en el LBA, incluso en los pacientes asintomáticos y sin alteraciones radiográficas ${ }^{16}$. En un estudio en cinco mujeres con cuadro clínico-radiográfico sugerente de OP post radioterapia se realizó LBA reportando recuentos celulares diferenciales de $37 \pm 6 \%$ linfocitos, $11 \pm$ $10 \%$ neutrófilos, $49 \pm 15 \%$ macrófagos, y 1,9 \pm $3 \%$ de eosinófilos ${ }^{6}$, confirmando la existencia de linfocitosis y disminución del recuento de macrófagos comparado con sujetos sanos.

Crestani y cols. ${ }^{12}$, tras describir a los quince pacientes iniciales, propusieron los siguientes criterios para el diagnóstico de OP secundaria a radioterapia en pacientes con cáncer de mama: 1) Radioterapia mamaria por cáncer en los últimos 12 meses; 2) Síntomas generales o respiratorios de al menos dos semanas de evolución; 3) Compromiso pulmonar en la radiografía o tomografía de tórax fuera de la zona irradiada y, 4) Ausencia de otra causa específica. Este último punto cobra mayor relevancia al considerar la amplia gama 
de diagnósticos diferenciales, los cuales superan en incidencia a la OP. Se debe tomar especial consideración en descartar las sobreinfecciones pulmonares bacterianas (incluyendo las micobacterias) y fúngicas y considerar la $\mathrm{OP}$ asociada a medicamentos, sobretodo a agentes quimioterapéuticos comúnmente utilizados en el cáncer de mama, como ciclofosfamida, doxorrubicina y metotrexato.

No se han identificado buenos predictores de OP post radioterapia, pero la edad sobre 50 años (OR: 8,8; IC95\% 1,16-67,76) y el tratamiento anti-hormonal concomitante (OR: 3,05; IC95\% $1,09-8,54)$ son factores de riesgo ${ }^{10}$.

Los mecanismos fisiopatológicos involucrados en la OP secundaria a radioterapia son aún desconocidos. A diferencia de la neumonitis actínica, donde el daño sería ocasionado por efecto directo de la radioterapia ${ }^{17}$, algunos autores ${ }^{18}$ postulan teorías de procesos autoinmunes gatillados por la radioterapia tangencial al pulmón ${ }^{16,18}$. Existen muy escasos reportes de OP secundaria a radioterapia torácica por otras neoplasias ${ }^{19}$, sin poder dilucidar el por qué.

El tratamiento esteroidal sistémico se emplea en la mayoría de los casos, describiendo mejorías precoces dramáticas ${ }^{10}$, tanto de los síntomas clínicos $^{12,14}$ como de los infiltrados radiográficos pulmonares, incluso con sólo dos semanas de tratamiento en algunos $\operatorname{casos}^{12}$. Los tratamientos abreviados de 4-5 meses se asocian a mayor tasa de recidiva ${ }^{9}$, siendo común que estas se presenten en los períodos de reducción de la dosis de la droga o tras su suspensión. El tiempo promedio de tratamiento empleado es de un año ${ }^{10,12}$. La recidiva ha sido descrita en 50 a $80 \%$ de los pacientes, sin embargo, en estos casos se han empleado dosis bajas de esteroides o el tiempo durante el cuál han sido utilizados ha sido breve ${ }^{10,18}$. Se han reportado casos puntuales en los que sólo se ha realizado tratamiento sintomático y observación de la evolución, sin emplear esteroides sistémicos, con respuesta clínica favorable en plazos de tres meses ${ }^{15,20}$. El pronóstico a largo plazo de esta condición es excelente, con tasas de recaída de $6 \%$ en el curso de cinco años ${ }^{5}$, no existiendo reportes de mortalidad asociada a esta complicación de la radioterapia.

En conclusión, el caso reportado ejemplifica los cuadros de OP asociada a radioterapia en pacientes con cáncer de mama, en los que el cuadro clínico orienta hacia una posible complicación pulmonar, que se condice con imágenes radiográficas de condensación que comprometen incluso áreas pulmonares alejadas de los campos de radiación, y que tienden a migrar en las imágenes radiológicas secuenciales. El tiempo entre el inicio de los síntomas hasta el diagnóstico definitivo del cuadro tarda en promedio 18 meses según lo reportado en la literatura ${ }^{12}$, lo que nos permite destacar la importancia de esta comunicación para contribuir a ampliar el diagnóstico diferencial a considerar en pacientes que presentan síntomas sistémicos y respiratorios inespecíficos asociado a infiltrados pulmonares migratorios tras radioterapia por cáncer de mama.

\section{Referencias}

1. Epler GR, Colby TV, McLoud TC, Carrington CB, Gaensler EA. Bronchiolitis obliterans organizing pneumonia. N Engl J Med 1985; 312: 152-8.

2. Colby TV. Pathologic aspects of bronchiolitis obliterans organizing pneumonia. Chest 1992; 102: 38S-43S.

3. American Thoracic Society/European Respiratory Society. International multidisciplinary consensus classification of the idiopathic interstitial pneumonias. Am J Respir Crit Care Med 2002; 165: 277-304.

4. Clarke M, Collins R, Darby S, Davies C, Elphinstone P, Evans E, et al. Early Breast Cancer Trialists' Collaborative Group (EBCTCG). Effects of radiotherapy and of differences in the extent of surgery for early breast cancer on local recurrence and 15-year survival: An overview of the randomised trials. Lancet 2005; 366: 2087-106.

5. Ogo E, Komaki R, Fujimoto K, Uchida M, Abe T, Nakamura $\mathrm{K}$, et al. A survey of radiation-induced bronchiolitis obliterans organizing pneumonia syndrome after breast-conserving therapy in Japan. Int J Radiat Oncol Biol Phys 2008; 71: 123-31.

6. Toma CL, Serbescu A, Alexe M, Cervis L, Ionita D, Bogdan MA. The bronchoalveolar lavage pattern in radiation pneumonitis secondary to radiotherapy for breast cancer. Maedica 2010; 5: 250-7.

7. Crestani B, Kambouchner M, Soler P, Crequit J, Brauner M, Battesti JP, et al. Migratory bronchiolitis obliterans organizing pneumonia after unilateral radiation therapy for breast carcinoma. Eur Respir J 1995; 8: 318-21.

8. Bayle JY, Nesme P, Béjui-Thivolet F, Loire R, Guérin JC, Cordier JF. Migratory organizing pneumonitis "primed" by radiation therapy. Eur Respir J 1995; 8: 322-6.

9. Takigawa N, Segawa Y, Saeki T, Kataoka M, Ida M, 
Kishino D, et al. Bronchiolitis obliterans organizing pneumonia syndrome in breast-conserving therapy for early breast cancer: radiation-induced lung toxicity. Int J Radiat Oncol Biol Phys 2000; 48: 751-5.

10. Katayama N, Sato S, Katsui K, Takemoto M, Tsuda T, Yoshida A, et al. Analysis of factors associated with radiation-induced bronchiolitis obliterans organizing pneumonia syndrome after breast-conserving therapy. Int J Radiat Oncol Biol Phys 2009; 73: 1049-54.

11. Miwa S, Morita S, Suda T, Suzuki K, Hayakawa H, Chida $\mathrm{K}$. The incidence and clinical characteristics of bronchiolitis obliterans organizing pneumonia syndrome after radiotherapy for breast cancer. Sarcoidosis Vasc Diffuse Lung Dis 2004; 21: 212-8.

12. Crestani B, Valeyre D, Roden S, Wallaert B, Dalphin JC, Cordier JF. Bronchiolitis obliterans organizing pneumonia syndrome primed by radiation therapy to the breast. Am J Respir Crit Care Med 1998; 158: 1929-35.

13. Arbetter KR, Prakash UB, Tazelaar HD, Douglas WW. Radiation induced pneumonitis in the "nonirradiated" lung. Mayo Clin Proc 1999; 74: 27-36.

14. Kano A, Ujita M, Kobayashi M, Sunakawa Y, Shirahama J, Harada T. Radiographic and CT features of radiation- induced organizing pneumonia syndrome after breastconserving therapy. Jpn J Radiol 2012; 30: 128-36.

15. Ogo E, Komaki R, Abe T, Uchida M, Fujimoto K, Suzuki G. The clinical characteristics and non-steroidal treatment for radiation-induced bronchiolitis obliterans organizing pneumonia syndrome after breast-conserving therapy. Radiother Oncol 2010; 97: 95-100.

16. Martín C, Romero S, Sánchez-Payá J, Massuti B, Arriero JM, Hernández L. Bilateral lymphocytic alevolitis: a common reaction after unilateral thoracic radiation. Eur Respir J 1999; 13: 727-32.

17. Logan PM. Thoracic manifestations of external beam radiotherapy. Am J Roentgenol 1998; 171: 569-77.

18. Prakash U. Radiation induced injury in the "nonirradiated" lung. Eur Respir J 1999; 13: 715-7.

19. Hamanishi T, Morimatu T, Oida K, Kori Y, Taguchi Y, Tanaka E. Occurrence of BOOP outside radiation field after radiation therapy for small cell lung cancer. Nihon Kokyuki Gakkai Zasshi 2001; 39: 683-8.

20. Fahim A, Campbell AP, Hart SP. Bronchiolitis obliterans organising pneumonia: a consequence of breast radiotherapy. BMJ Case Rep 2012; doi:10.1136/ bcr.10.2011.4987. 